\title{
Humanoids, Brain and Cognitive Sciences
}

\author{
Giulio Sandini \\ Department of Robotics, Brain and Cognitive Sciences \\ Italian Institute of Technology and \\ LIRA-Lab, University of Genoa
}

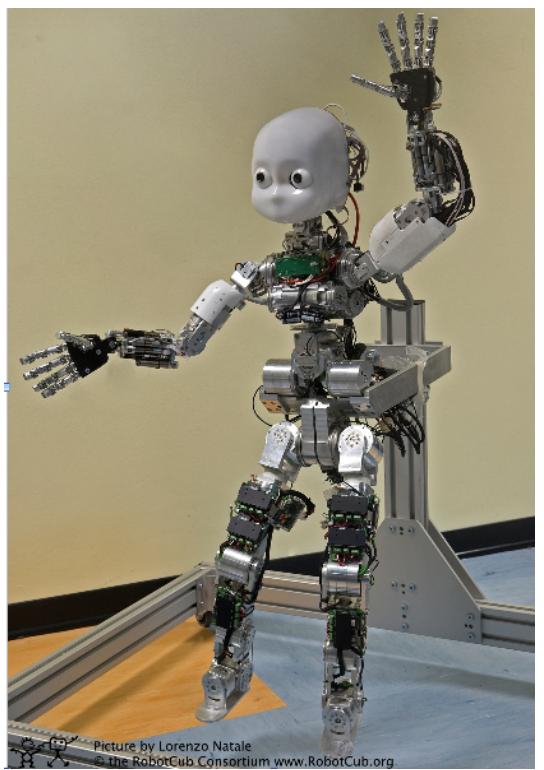

In the talk I will discuss how research on humanoid robots, cognition and brain sciences can be seen as parts of a multidisciplinary, coordinated effort aimed at advancing knowledge on the foundation of human intelligence and at developing new, human-centered technologies. The rationale stems from the observation that developing human-like intelligence in artificial systems with human-like morphology (humanoids) requires to address the same questions cognitive neuroscientists are asking through experimental investigations. Conversely understanding human intelligence from all its multifaceted perspective can take advantage of the realistic simulation allowed by the physical implementation of hardware models. Within this framework I will present results of projects ongoing at the Department of Robotics, Brain and Cognitive Sciences of IIT in the areas of humanoid cognition, robotic rehabilitation and motor learning, multimodal sensory integration and brain machine interface. 